\title{
DOES SERUM BRAIN NATRIURETIC PEPTIDE (BNP) TOGETHER WITH A NOVEL PDA SCORE IMPROVE PDA TREATMENT SELECTION?
}

Y. Elsayed, S. Dakshinamurti, R. Soni, I. Buffo, M.M. Seshia

Pediatrics, University of Manitoba, Winnipeg, MB, Canada

Background: The determination of whether or not a PDA is hemodynamically significant, requiring treatment, poses a dilemma to the clinician. BNP measurement has been reported to be useful in the diagnosis of a hemodynamically significant PDA (HSPDA) in preterm infants.

Objectives: To determine whether serum Brain Natriuretic Peptide (BNP), together with a high PDA score measured between 48-72 hours of life, predict a (HSPDA) requiring closure in infants $<31$ weeks GA.

Study design: This prospective, blinded cohort study was conducted on Infants $<31$ weeks GA, admitted August 2010 to February 2011, to NICU in Winnipeg, Canada, had, following parental consent, bedside serum BNP assay, echocardiogram and a novel PDA score determined between 48-72 hours postnatally, blinded from the treating team. BNP was correlated with echocardiographic parameters and PDA score. Reference standard for HSPDA was PDA diameter $>1.5 \mathrm{~mm}$ by ECHO with left to right non-restrictive shunt.

Results: Thirty four of ninety eligible neonates were studied. HSPDA was present in 17 . Mean $( \pm$ SD) gestational age (weeks) for HSPDA group was 26.7 \pm 1.2 , non-HSPDA group 28.6 $\pm 1.1(\mathrm{p}<.000$ ); birth weight (g) HSPDA group was $897 \pm 16$, non-HSPDA group $1270 \pm 27(\mathrm{p}<.000$ ). Median (IQ range) PDA score for HSPDA group was $15(9,14.5)$, non-HSPDA group $3(2,3.5)(\mathrm{p}<.000)$. Median (IQ range) BNP for HSPDA group was 320(120,671)pg/ml, non HSPDA group 16(6,34)pg/ml(p<.000). For HSPDA, sensitivity, specificity, positive and negative predictive values for both PDA score and BNP were $94 \%, 100 \%, 100 \%$, $94 \%$, respectively.

Conclusion: Both BNP and PDA score predict HSPDA requiring treatment. Cut off values were 90pg/ml, score $>7$ respectively. 